\title{
An Ultraviolet-Visible (UV) Photometry System Based on the PDMS-based Microfluidic Chip
}

\author{
Changhua Xiang ${ }^{1, \mathrm{a}}$, Ning Yang ${ }^{1,2, \mathrm{~b}}$, Rongbiao Zhang ${ }^{1, \mathrm{c}}$, Jianjiang Guo ${ }^{1, \mathrm{~d}}$ and Hu Huang ${ }^{3, \mathrm{e}}$ \\ ${ }^{1}$ School of Electrical and Information Engineering, Jiangsu University, Zhenjiang, PR China \\ ${ }^{2}$ Institute of Agricultural Engineering, Jiangsu University, Zhenjiang, PR China \\ ${ }^{3}$ Department of Biomedical Engineering, University of Minnesota, Minneapolis, United States

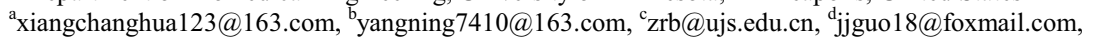 \\ 'huhuang:hwangtiger@gmail.com
}

\begin{abstract}
In order to avoid a problem remains with the low accuracy and poor portability of the photometry system, the system based on the photometry method and microfluidic chip technology was built. As the characteristics of cheap, solid and good transmission, Polydimethylsiloxane (PDMS) was chosen as the material of the designed chip in the paper. To the designed UV photometry system, the light-emitting diode with the wavelength of $580 \mathrm{~m}$ is chosen as the light source. The experimental result indicates that there is no significant deviation between the designed UV photometry system and the conventional immuneturbidimetric assay, the correlation coefficient is 0.95 obtained by adopting the linear regression analysis. The linearity of the designed UV photometry system based on the PDMS-based microfluidic chip has increased by $17.3 \%$ in comparison with the system based on the silicon-based microfluidic chip.

Keywords. Polydimethylsiloxane (PDMS), photometry, microfluidic chip, agglutination
\end{abstract}

\section{Introduction}

The photometry method is a qualitative and quantitative detection technology to substances, which adopts absorption spectra and emission spectra that are peculiar to substances [1]. It has some advantages (i.e., such as simple instruments, easy operation and rapid detection, etc.), which has a wide range of applications in science, engineering, agriculture, medicine and so on. The microfluidic chip technology is a platform that integrates one or several laboratory functions on a single chip of only millimeters to a few square centimeters to achieve automation and high-throughput screening [2]. The combination of the microfluidic chip technology and the photometry method has widely been applied in science, engineering, agriculture, medicine. Pinto etc. collected the samples of the natural water within 1 hour to detect the content of copper in water on the platform of the microfluidic chip by using the photometry method. The detection limit could reach up to $4.6 \mu \mathrm{g} / \mathrm{L}$ and the average error is $5.32 \%$ [3]. Compare with other detection methods, photometry method based on the microfluidic chip technology can directly detect the samples without adding additional reagents, and it has the characteristics of high feasibility and simple operation. So the UV photometry system based on the microfluidic chip is built in the paper. However, the downside of the system is low metering accuracy. So it is necessary to build a portable photometric system with high precision.

For the designed microfluidic chip, there are many kinds of materials to fabricate the microfluidic chip including silicon, paper and PDMS. The process to prepare the microfluidic chip by the silicon is the ripest one [4]. However, the cost of silicon is relatively high which limits the application in the fabrication and application of microfluidic chip [5]. Paper is relatively cheap material compare to the silicon. However, paper is fragile which also limits the application too [6]. PDMS $\left(\left(\mathrm{C}_{2} \mathrm{H}_{6} \mathrm{OSi}\right)_{n}\right)$ has appeared in the last few years [7]. As the characteristics of cheap, solid and good transmission, PDMS is chosen as the material of the designed chip in the paper. Based on the designed chip, a portable photometric system with high precision is built.

\section{The material of chip and corresponding fabrication method}

There are many kinds of materials to fabricate the microfluidic chip like silicon, paper and PDMS. The silicon is the earliest in fabricating the microfluidic chip as the productions process of silicon is the most mature compare to paper and PDMS. However, there are also some disadvantages to the silicon for example the cost of silicon is relatively high which limits its application in lab-on-a-chip fabrication. Paper is relatively cheap compare to the silicon. However, the paper has a serious unfairness--the paper is fragile, which also limits the application in the field of the chip's fabrication. PDMS has appeared in the last few years, it has widely applied in fabricating the microfluidic chip as it has the characteristics of cheap, 
solid and good transmission. So, PDMS is regarded as the material to fabricate the microfluidic chip in the paper. Table 1 is the characters of three different materials.

Table 1. The characters of three different materials.

\begin{tabular}{|c|c|c|c|}
\hline Character & Cost & Transparency & $\begin{array}{c}\text { Fragile } \\
\text { article }\end{array}$ \\
\hline Silicon & High & Bad & Yes \\
\hline Paper & Low & Bad & Yes \\
\hline PDMS & Low & Good & No \\
\hline
\end{tabular}

Considering the experimental condition and fabricating cost, the imprinting method is used to fabricate the microfluided chip, the fabrication processes are shown in Figure 1. During the processes, the imprinting method also needs a mould that should be made previously, such as silicon or quartz mould. The substrate and mould are pressed by the stamp technique. In other words, substrate and mould are bonded together by using appropriate force at an appropriate time. Then, the desired shape has been formed on the substrate. The final step is linkage. To protect substrate, the package is needed after all steps are finished. With the help of Suzhou Daya Electronics Co., LTD, the PDMS-based microfluidic chip is fabricated successfully.

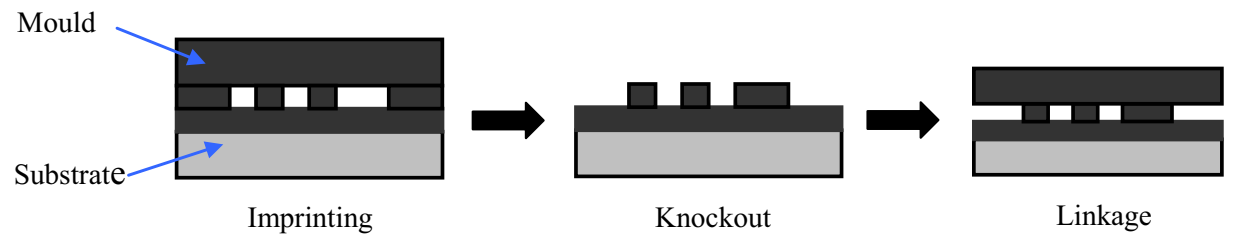

Fig.1. The production process of microfluidic chip.

\section{The comprehensive system design}

Design of the microfluidic chip. Figure 1 is the production process of the designed microfluidic chip. Figure 2 (a) is top view of the designed microfluidic chip and Fig. 2 (b) is elevation view of the designed microfluidic chip. In the Figure 2 (a), $a$ is the $\mathrm{Y}$-shaped sampling zone, $b$ is the mixing zone, $c$ is the reaction zone and $d$ is the photoelectric detection zone. The reagents are placed in the injection pumps to prepare for mixing and detecting. Then, reagents are injected into the microfluidic chip from the sampling zone $a$ under the injection pumps. As reagents flow into the mixing $b$, the adequate mixing happens in the mixing zone $b$. In the paper, there is an immunization agglutination reaction between the sensitized latex reagent and the corresponding serum in the reaction zone $c$. Some time is needed before the chemical reaction has set off sufficiently. In the experiment, the visible agglutinators begin to appear as the chemical reaction goes on, and the corresponding photometric detection is needed at the photoelectric detection zone $d$ to determine the extent of immunization agglutination reaction. The designed microfluidic chip is transparent as the material of the designed microfluidic chip is PDMS, so it is possible to determine extent of immunization agglutination reaction at the photoelectric detection zone $d$ by using the transmission characteristic of light.

To the mixing zone $b$, the structure of inner rib is designed to achieve the intensive mixing between different liquids. Supposing that the titer of two different liquids is 1 and 0 respectively, the effect of reagents mixing could be simulated by the simulation software of COMSOL Multiphysics 5.1. The simulations reveal that the designed structure has a good mixing effect and the titer is close to 0.5 at the outlet. So, the intensive mixing could be achieved when liquids flow past the mixing zone $b$.

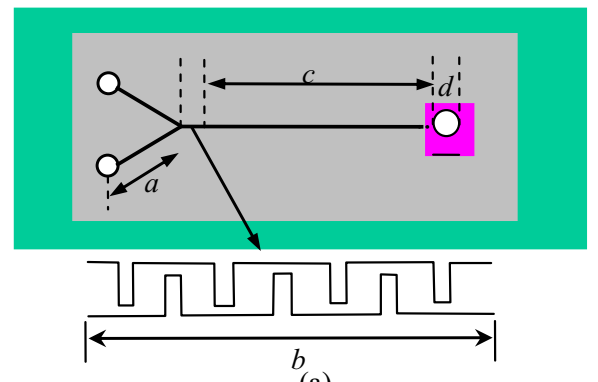

(a)

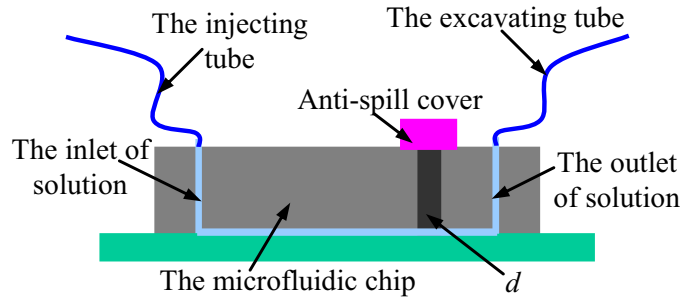

(b)

Fig.2. The schematic diagram of designed microfluidic chip. 
(a) Top view of the designed microfluidic chip.

(b) Elevation view of the designed microfluidic chip.

Optimal absorbance wavelength of the immune agglutination. The optimal wavelength is the wavelength that can reflects the optimal relationship between the solution concentration and light absorption, which is also called the resonant frequency light-wave. During the experiment, the light-emitting devices connect with the precision source-ocean optics HL-2000-HP-FHSA, the detection devices connect with ocean optics USB2000 spectrograph. The rheumatoid factor positive serums, with the titers of $20 \mathrm{IU} / \mathrm{mL}, 30 \mathrm{IU} / \mathrm{mL}, 40 \mathrm{IU} / \mathrm{mL}, 50 \mathrm{IU} / \mathrm{mL}$, and $60 \mathrm{IU} / \mathrm{mL}$, will have a reaction with the sensitized latex reagent respectively.

Agglutination reactions produce visible aggregates of antibody-antigen complexes when antibodies or antigens are conjugated to a carrier. Carriers used in agglutination methods could be artificial or biological. Table 2 is the absorption value of the designed UV photometry system varies according to the wavelength range from $500 \mathrm{~nm}$ to $600 \mathrm{~nm}$.

Table 2. The absorption value of the designed system varies according to the wavelength.

\begin{tabular}{|c|c|c|c|c|c|c|c|c|c|c|c|}
\hline $\begin{array}{c}\text { Absorbance Wavelength } \\
(\mathrm{Abs})\end{array}$ & 500 & 510 & 520 & 530 & 540 & 550 & 560 & 570 & 580 & 590 & 600 \\
\hline Titer (IU/mL) & 0.12 & 0.13 & 0.135 & 0.139 & 0.142 & 0.150 & 0.156 & 0.159 & 0.160 & 0.154 & 0.150 \\
\hline 20 & 0.18 & 0.193 & 0.201 & 0.209 & 0.214 & 0.225 & 0.23 & 0.237 & 0.240 & 0.228 & 0.220 \\
\hline 30 & 0.27 & 0.28 & 0.288 & 0.297 & 0.300 & 0.305 & 0.308 & 0.312 & 0.311 & 0.302 & 0.290 \\
\hline 40 & 0.300 & 0.312 & 0.320 & 0.330 & 0.339 & 0.350 & 0.360 & 0.369 & 0.377 & 0.365 & 0.350 \\
\hline 50 & 0.360 & 0.423 & 0.420 & 0.432 & 0.434 & 0.450 & 0.462 & 0.475 & 0.48 & 0.468 & 0.450 \\
\hline 60 & &
\end{tabular}

From the table 2, the result has revealed that the absorbance values increases with the detection wavelength between $500 \mathrm{~nm}$ and $580 \mathrm{~nm}$. The absorbance values decreases with the detection wavelength when the wavelength is greater than $580 \mathrm{~nm}$. So, the wavelength of $580 \mathrm{~nm}$ is the optimal absorbance wavelength. When the detection wavelength is $580 \mathrm{~nm}$, the absorbance reaches maximum no matter what the titers of the positive serums are.

Design of detection circuit. The detection circuit is built by the designed microfluidic chip, the light-emitting diode and the photodiode. To enhance the sensitivity and accuracy of detection circuit, the detection circuit is built in a vertical way which is shown in Figure 3.

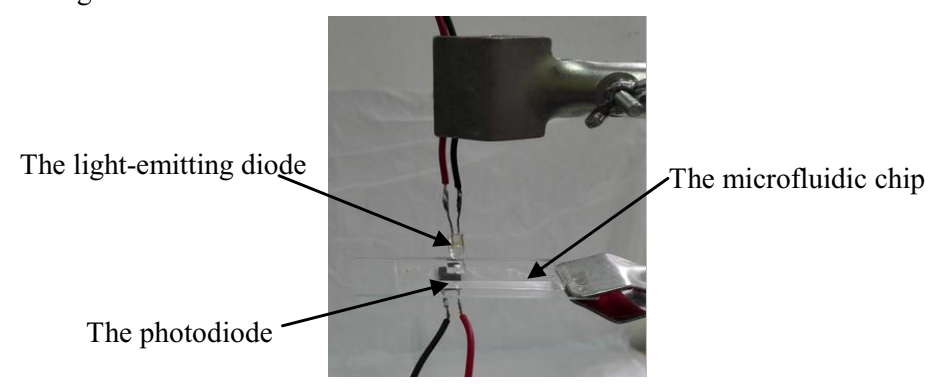

Fig.3. The physical picture of optical path detection circuit.

During the detecting, the light-emitting diode sends out the light of wavelength $580 \mathrm{~m}$, and the photodiode absorbs the light emitted by light-emitting diode. There is a lid over the photoelectric detection zone $c$ for preventing the overflow of liquids, and the glass base under the chip is regard as base. The surface of PDMS substrate, anti-spill lid and glass base are dealt with by plasma oxidation method in advance. Then the PDMS substrate, anti-spill lid and glass base are recombined to enable the seam to be more solid and stable. As the thickness of chip is $5 \mathrm{~mm}$, so the optical path length of $5 \mathrm{~mm}$ could be obtained.

Overall Experimental Platform Construction. Figure 4 is a picture of the designed photometry system. To ensure sufficient reaction of the agglutination reaction, the indoor temperature should be kept at $37^{\circ} \mathrm{C}$. The microfluidic chip is placed between the light-emitting diode and the photodiode. The microfluidic chip connects with the sampling-drive pump (the velocity of pump is $0.098 \mu \mathrm{m} / \mathrm{step}$, and the accuracy of CVs is less than $1 \%$ ) through the pipelines. The microfluidic chip is placed between the light-emitting diode and the photodiode. The microfluidic chip connects with the sampling-drive pump (the velocity of pump is $0.098 \mu \mathrm{m} / \mathrm{step}$, and the accuracy of CVs is less than $1 \%$ ) through the pipelines. 


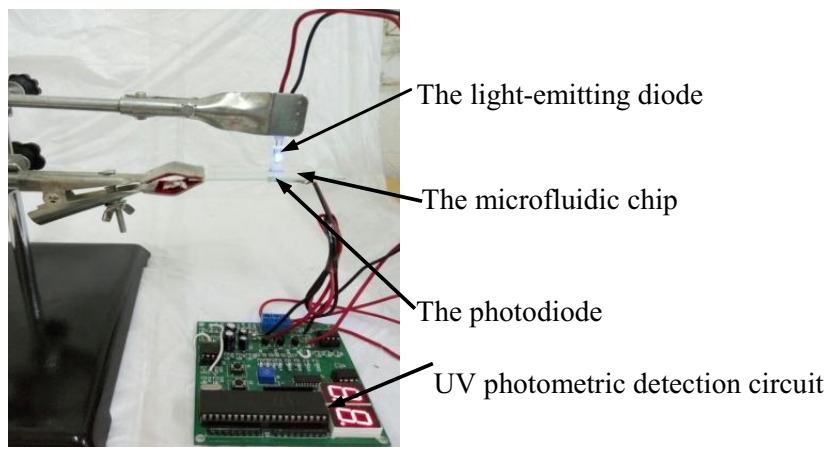

Fig.4. The designed UV photometry detection system based on microfluidic chip.

The sensitized latex reagent (pure and surface inactive polystyrene latex particles with the diameter of $50 \mathrm{~nm}$, and it is provided by Shanghai Jiemen Bio-tech co.) is injected into one syringe, the positive serums sample with different titers of the rheumatoid factor is injected into the other syringe. The detection circuit connects to $-10 \mathrm{~V}$ and $+5 \mathrm{~V}$ power supplies, the designed system adopts the real time controlling MCS-51 single-chip microprocessor as centre controller, and the result of absorbance detection could be displayed by the LED numerical code tube.

\section{Experimental results}

In the experiment, the wavelength of $580 \mathrm{~nm}$ is chosen as the optimal wavelength, PDMS is chosen as the material of the chip and the microfluidic chip is fabricated by adopting the imprinting method. The PH value of the sensitized latex reagent is 7.5 , the velocity of $0.08 \mathrm{~mm} / \mathrm{s}$ is chosen as the injection rate and the experimental temperature of the photometry system is $37{ }^{\circ} \mathrm{C}$. After the basic parameters have been determined, the photometry detection method and the precise instrument are carried out. The titers of positive serums are $20 \mathrm{IU} / \mathrm{mL}, 25 \mathrm{IU} / \mathrm{mL}, 30 \mathrm{IU} / \mathrm{mL}, 35 \mathrm{IU} / \mathrm{mL}, 40 \mathrm{IU} / \mathrm{mL}, 45$ $\mathrm{IU} / \mathrm{mL}, 50 \mathrm{IU} / \mathrm{mL}, 55 \mathrm{IU} / \mathrm{mL}$, and $60 \mathrm{IU} / \mathrm{mL}$. From the Figure 5, we may draw a conclusion that the correlation coefficient is 0.95 obtained by adopting the linear regression analysis. So, there is no significant deviation between the designed UV photometry system and the conventional immuneturbidimetric assay. The linearity of photometry system based on the silicon-based microfluidic chip is 0.81 [8], so the linearity of photometry system based on the PDMS-based microfluidic chip has increased by $17.3 \%$ in comparison with the system based on the silicon-based microfluidic chip.

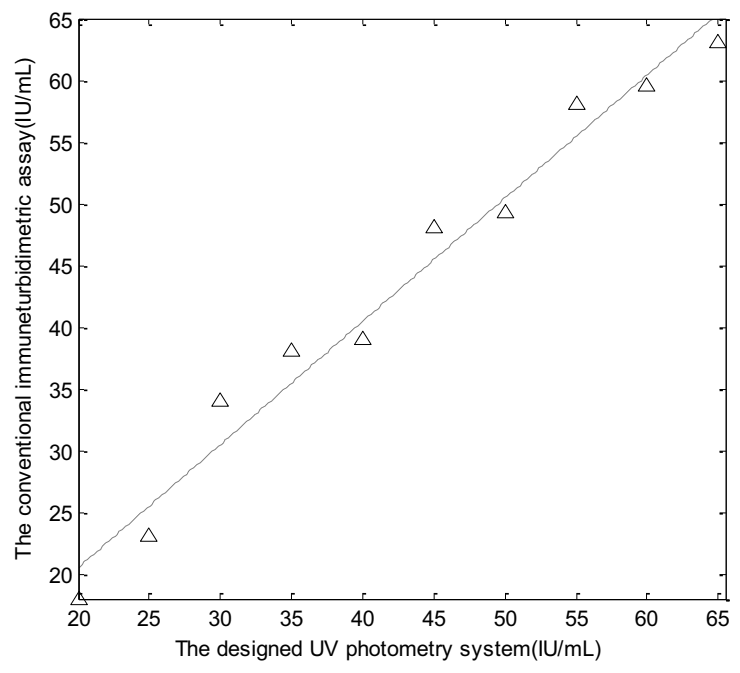

Fig.5. Regression analysis for the two different detection methods.

\section{Conclusions}

In the paper, a portable UV photometry system based on microfluidic chip is designed and the material of microfluidic chip is PDMS. The photometry system is a combination between the microfluidic chip technology and photometry detection technology. The microfluidic chip is fabricated by the imprinting method and the light-emitting diode which sends out 
$580 \mathrm{~m}$ light is selected as one part of the detection circuit. The designed UV photometry system based on the microfluidic chip is built with the light-emitting diode, the photodiode, the detection circuit and other devices. Finally, the correlative analysis experiment between the photometry detection method and conventional precise instrument is carried out. The experimental result indicates that there is no significant deviation for the designed photometry system compares with the precise instrument, the correlation coefficient is 0.95 obtained by adopting the linear regression analysis. The linearity of the designed UV photometry system based on the PDMS-based microfluidic chip has increased by $17.3 \%$ in comparison with the system based on the silicon-based microfluidic chip. So, PDMS is a better material for fabricating the microfluidic chip and the designed UV photometry system has the advantage of portability.

\section{Acknowledgements}

Supported by the Priority Academic Program Development of Jiangsu Higher Education Institutions(PAPD), Natural Science Foundation of Jiangsu Province(BK20140550), China Postdoctoral Science Foundation (2015T80512), Changzhou Sci\&Tech Program(CE20155054)and Research and Development Plan of Zhenjiang to Modern Agriculture(NY2015026).

\section{References}

1. M.I. Leong, S.D. Huang. Dispersive liquid-liquid microextraction method based on solidification of floating organic drop combined with gas chromatography with electron-capture or mass spectrometry detection. J. Chromatogr. A, Vol. 1211(2008), p. 8-12.

2. G.M. Whitesides. The origins and the future of microfluidics. Nature, Vol. 442(2006), p. 368-373.

3. J.J. Pinto, C. Moreno, M. García-Vargas. A very sensitive flow system for the direct determination of copper in natural waters based on spectrophotometric detection. Talanta, Vol. 64(2004), p.562-565.

4. R. Kangning, Z. Jianhua, W. Hongkai. Materials for Microfluidic Chip Fabrication. Accounts Chem. Res., Vol. 11(2013), p.2396-2406.

5. A. Mata, A.J. Fleischman, S. Roy. Characterization of Polydimethylsiloxane (PDMS) Properties for Biomedical Micro/Nanosystems. Biomed. Microdevice, Vol. 4(2006), P.281-293.

6. Q.H. He, C.C. Ma, X.Q. Hu, H.W. Chen. Method for fabrication of paper-based microfluidic devices by alkylsilane self-assembling and UV/O 3 -patterning. Anal. Chem., Vol. 85(2013), P.1327- 1331.

7. M.A. Eddings, M.A. Johnson, B.K. Gale. Determining the optimal PDMS-PDMS bonding technique for microfluidic devices. J. Micromech. Microeng., Vol. 18(2008), P.1171-1185.

8. J.W. Lee, R. Investigation of thermal vibration correlation of silicon lattice atoms by ion scattering. Nucl. Instrum. Meth. B, Vol. 232(2005), p.285-289. 